\title{
US puts large-scale AIDS vaccine trials on ice as 'premature'
}

Washington. Plans for large-scale clinical trials of preventive AIDS vaccines in the United States have been put on ice following the ruling last week of a key advisory panel that the candidate gp120 vaccines are not ready for such tests.

The formal decision on whether the trials should proceed rests with Anthony Fauci, director of the National Institute of Allergy and Infectious Diseases (NIAID). But immediately after his AIDS Research Advisory Committee (ARAC) came out against the trials last Friday, he said they would not go ahead.

The advisory panel's verdict overturns a recommendation in April of NIAID's Vaccine Working Group, which wanted to test two candidate vaccines and a placebo on 4,500 people at high risk of HIV infection. But Fauci denies that the decision is a setback for AIDS vaccine research.

Representatives of Genentech and Biocine, the developers of the two candidate vaccines, were devastated by the decision and angry at the way it was reached. But it has been welcomed by AIDS activists, who had doubted the value of the trials.

The decision leaves AIDS with no immediate prospects for either a preventive vaccine, a therapeutic vaccine, or a satisfactory drug therapy. It will also reinforce the recent and widely endorsed call by Bernie Fields of Harvard Medical School (see Nature 369, 95 ; 1994) for AIDS research to return to basics, and concentrate on the pathogenesis of the disease.

After eight hours of discussion, the advisory panel voted to "continue the current gp120 program and the development of other candidates currently under study", and to "proceed with expanded clinical trial evaluation when another concept and/or compelling data from current or other studies are available".

There were 32 votes cast in favour of this wording, four abstentions and none against, enabling Fauci to claim a "unanimous" vote. But in practice the panel was deeply divided, with those supporting a large trial caving in as it became clear that they were in a minority.

Rod Hoff of NIAID's division of AIDS told the panel that the 4,500 -strong trial - proposed by the working group as a half-way house to a full-scale, Phase 3 efficacy trial in-

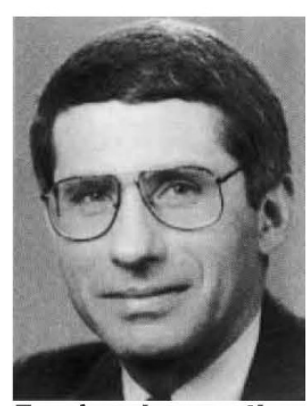

Fauci: welcomes the decision. volving 9,000 people - would show whether the vaccines were more than 60 per cent effective or less than 30 per cent effective. But he said that a larger follow-up would be required if their effectiveness was between those levels.

Although the candidate vaccines have not been formally tested for efficacy, a number of patients have become HIV positive during safety trials, and many panel members felt that the vaccine was unlikely to be effective enough to make the 4,500strong trial worthwhile.

The meeting also heard evidence that recruitment for the trial would be difficult, as volunteers were likely to withdraw when they were informed that the vaccine, al- though safe, was based on part of the virus, and that some would test "false positive" for HIV for a short time during trial.

In a decisive intervention, Yung-chi Cheng of Yale University warned that a misdirected trial would damage both the future of vaccine research and public confidence. "I'm really trying to convince myself that this has some validity," he said. "[But] it doesn't have any merit on the information we have."

Working group member Susan ZollaPazner of New York Veterans Affairs Medical Center tried to rally support for its proposal of a 4,500-strong trial, saying that it was based on a more thorough examination of the scientific data than the 40 -member advisory panel had been able to undertake.

Searching for a compromise, Warren Winkelstein of the University of California at Berkeley called for a "new mechanism" which would "move things forward without saying that we have the answer to the epidemic". But panel members argued that a large-scale trial that was not a Phase 3 efficacy trial would confuse the public, and was likely to be ineffective in meeting other declared objectives, such as establishing the correlates of immunity of the disease.

The meeting moved quickly and somewhat chaotically to issue its recommendation, effectively killing the trial. Earlier, Fauci had told the meeting that if the trial proposal was abandoned, "there may be a chilling effect on our industrial partners, although this in itself is not an acceptable rationale for proceeding".

Fauci had promised himself some leeway in making a final decision after Friday's meeting. But in the event he wasted no time in endorsing the panel's advice. "I have accepted it," he said. "I have no option, and I agree with them."

Although the panel agreed on a statement stressing that their recommendation applies only to NIAID and the United States, the decision is likely to deal a hammer blow to prospects for large-scale vaccine trials in other countries.

Advocates of such trials will say that the presence of very high risk populations - for example in the sex industry in Thailand make trials more feasible there. But opponents are now likely to argue that the vaccines rejected by the United States are being "dumped" abroad.

The United States will return to the question of a trial either when some breakthrough is made on gp 120 , or when alternative vaccines are ready. Fauci says this could take between one and three years.

Colin Macilwain 